\title{
MAY-THURNER SYNDROME AS A DIFERENTIAL DIAGNOSIS OF RHEUMATOLOGICAL DISEASES IN PEDIATRICS
}

\author{
Teresa Cristina Martins Vicente Robazzi ${ }^{1, \star}$, Beatriz Oliveira Leão Carneiro ${ }^{1}$, Pedro Herrera Camargo ${ }^{1}$, Leandra Chaves Barros $^{1}$ \\ 1.Universidade Federal da Bahia, Salvador (BA), Brazil. \\ *Corresponding author: trobazzi.ufba@gmail.com
}

\section{BACKGROUND}

Iliac vein compression syndrome (IVCS), also known as May-Thurner or Cockett syndrome, occurs from compression of the left common iliac vein by the right common iliac artery. The main clinical manifestations are pain and edema in the left lower limb, or left iliofemoral deep vein thrombosis (DVT). The main risk factors for DVT are prolonged rest, pregnancy and surgery.

\section{CASE REPORT}

AES, female, 13 years old, with a history of chronic pain and edema in the left lower limb. Referred to a reference service in Pediatric Rheumatology for investigation of a possible diagnosis of scleroderma. On examination, she presented diffusely significant asymmetry between the lower limbs, without signs of arthritis and without other alterations on physical examination. Inflammatory evidence unchanged. Computed tomography angiography of the limbs showed the presence of extrinsic compression of the left common iliac vein by the right common iliac artery, compatible with the diagnosis of SCVI or May-Thurner syndrome. After this diagnosis, the patient. Clinical conditions such as the one reported require early diagnosis and intervention. For SCVI, when the patient is symptomatic, surgical treatment is indicated, with the aim of treating symptoms related to venous hypertension and preventing sequelae of venous occlusive disease.

\section{CONCLUSION}

For pediatric rheumatologists, it is important to make the differential diagnosis of pain and edema in limbs with vascular diseases, considering the need for early intervention to ensure better outcomes and reduce associated morbidity and mortality. Despite their rarity, these diagnostic possibilities should also be considered in the pediatric age group, not just in adult patients.

\section{KEYWORDS}

May-Thurner syndrome, Iliac disease, Rheumatological disease, Limb pain, Pediatric. 